\title{
Correlation Between Drug Sensitivity Profiles of Ex Vivo Expanded Circulating Tumor Cells and Clinical Treatment Response in Pancreatic Ductal Adenocarcinoma Patients
}

\author{
Yuan-Hung Wu \\ Taipei Veterans General Hospital \\ Yi-Ping Hung \\ Taipei Veterans General Hospital \\ Nai-Chi Chiu \\ National Yang Ming Chiao Tung University \\ Rheun-Chuan Lee \\ National Yang Ming Chiao Tung University \\ Chung-Pin Li \\ National Yang Ming Chiao Tung University \\ Yee Chao \\ Taipei Veterans General Hospital \\ Yi-Ming Shyr \\ National Yang Ming Chiao Tung University \\ Shin-E Wang \\ National Yang Ming Chiao Tung University \\ Shih-Chin Chen \\ National Yang Ming Chiao Tung University \\ Sheng-Hsuan Lin \\ National Yang Ming Chiao Tung University \\ Yi-Hsuan Chen \\ Taipei Medical University \\ Yu-Mei Kang \\ Taipei Veterans General Hospital \\ Shih-Ming Hsu \\ National Yang Ming Chiao Tung University \\ Sang-Hue Yen \\ Taipei Veterans General Hospital \\ Jeng-You Wu \\ Taipei Municipal Wan-Fang Hospital
}




\section{Kuan-Der Lee}

Taipei Medical University Hospital

\section{Huey-En Tseng}

Taipei Medical University Hospital

\section{Jia-Ruey Tsai}

Taipei Medical University Hospital

\section{Ruey-Hsiang Tang}

Taipei Medical University Hospital

\section{Jeng-Fong Chiou}

Taipei Medical University

\section{Thierry Burnouf}

Taipei Medical University

\section{Yin-Ju Chen}

Taipei Medical University

\section{Peng-Yuan Wang}

Swinburne University of Technology

\section{Long-Sheng Lu ( $\sim$ Islu@tmu.edu.tw )}

Taipei Medical University https://orcid.org/0000-0002-4247-1981

\section{Research}

Keywords: pancreatic ductal adenocarcinoma, circulating tumor cells, liquid biopsy, personalized cancer medicine

Posted Date: August 31st, 2021

DOl: https://doi.org/10.21203/rs.3.rs-845754/v1

License: (c) (1) This work is licensed under a Creative Commons Attribution 4.0 International License. Read Full License 


\section{Abstract}

\section{Background}

Pancreatic ductal adenocarcinoma (PDAC) is highly aggressive and has poor prognosis. There are few biomarkers to inform treatment decisions, and collecting tumor samples for genomic or drug sensitivity testing is challenging.

\section{Methods}

Circulating tumor cells (CTCs) were prepared from the liquid biopsies of PDAC patients. These cells were subsequently expanded ex vivo to form CTC-derived organoid cultures, using a laboratory-developed biomimetic cell culture system. The CTC-derived organoids were tested for sensitivity to a PDAC panel of nine drugs, with tests conducted in triplicate, and a weighted cytotoxicity score (CTS) was calculated from the results. Clinical response to treatment in patients was evaluated using Response Evaluation Criteria in Solid Tumors (RECIST) version 1.1 criteria at the time of blood sampling and 3 months later. CTS was then correlated with clinical response, and analyzed using $2 \times 2$ contingency tables.

Results

A total of 41 liquid biopsies were collected from 31 patients, with $87.8 \%$ of liquid biopsies from patients with Stage 4 disease. CTC-derived organoid expansion was achieved in 3 weeks, with $87.8 \%$ culture efficiency. CTC-derived organoid cultures were positive for EpCAM staining and negative for CD45 staining in surface marker analysis. All patients had received a median of two lines of treatment prior to enrollment, and prospective utility analysis indicated significant correlation of CTS with clinical treatment response. Two representative case studies are also presented to illustrate the relevant clinical contexts.

\section{Conclusions}

In this study, CTCs were expanded from the liquid biopsies of PDAC patients with a high success rate. Drug sensitivity profiles from CTC-derived organoid cultures correlated meaningfully with treatment response. Further studies are warranted to validate the predictive potential for this approach.

Trial Registration

Taipei Medical University Hospital Protocol Record N201803020, registered on July 10, 2018; ClinicalTrials.gov Identifier: NCT04972461, retrospectively registered on July 22, 2021.

\section{Background}

Pancreatic ductal adenocarcinoma (PDAC) accounts for more than $85 \%$ of all pancreatic cancers, and is one of the most aggressive forms of solid malignancy currently known, with 5-year survival rates of around $10 \%$ [1]. At initial diagnosis, approximately $80 \%$ of patients have unresectable disease, and more 
than $50 \%$ have metastatic disease [2]. In such cases, systemic chemotherapy represents the standard of care, and progression-free survival (PFS) is usually limited to 3-6 months [3]. Unsurprisingly, the GLOBOCAN 2020 statistics rank PDAC as the seventh leading cause of cancer mortality worldwide [4], and considering such poor outcomes, there is an urgent need to improve the current approach to PDAC systemic treatment.

A promising approach that has seen success in the management of lung cancer [5] and breast cancer [6] is the use of surrogate biomarkers to assess and predict clinical response to treatment, thereby allowing patients to be treated with therapies that have the best chance of being effective. This approach is also applicable to PDAC, and a recent study found that somatic BRCA1 mutations and genetic defects in the homologous recombination pathway were predictive of better PFS in PDAC patients receiving maintenance therapy [7-9]. However, this approach has a key caveat in that tumor tissue is needed to assess biomarker status, but tumor biopsies or resection specimens are difficult to obtain from PDAC patients.

Liquid biopsies containing circulating tumor cells (CTCs) can serve as a source of tumor tissue that is relatively easy to obtain and is minimally invasive. Most studies in PDAC have focused on CTC enumeration, and higher CTC levels have been found to correlate with disease progression and poor treatment response $[10,11]$. However, recent studies in colorectal cancer $[12,13]$ have sought to isolate and expand CTCs ex vivo, in order to generate CTC-derived cultures that can be directly tested for sensitivity to a range of different anti-cancer treatments. The results of these studies are promising, but key obstacles remain, such as low culture efficiencies of less than $20 \%$ and high costs [14-16]. Discrepancies have also been reported between different methods of CTC detection and extraction [1416]. Despite these obstacles, a consistent, reliable, and cost-effective method of isolating and expanding CTCs from PDAC patients would be very useful for developing personalized treatment strategies, and can be expected to benefit patient outcomes.

In this study, we collected 41 liquid biopsies from 31 PDAC patients, and then proceeded to isolate and expand CTCs ex vivo in a laboratory-developed biomimetic cell culture system known as eSelect. The eSelect system does not use size elimination or epithelial marker capture methods, thereby preserving the heterogeneity of CTCs to ensure that CTC-derived organoid cultures are more representative of actual tumor conditions. These CTC-derived organoid cultures were assessed for sensitivity to a panel of nine anti-cancer drugs, and the results were correlated with clinical response in patients over a 3-month followup period. A previous study using the eSelect system showed good correlation between the drug sensitivity profiles of CTC-derived organoid cultures and clinical response to treatment in small cell lung cancer patients [17], and demonstrated the potential to reduce costs and time involved with culturing and testing. Here we showed that drug sensitivity results derived from CTC-derived organoids, calculated as a cytotoxicity score (CTS), correlated significantly with clinical outcomes in prospective utility analysis. Moreover, drug sensitivity results predicted the presence of rare but actionable genotypes in PDAC patients, and we present two illustrative case results to highlight the potential of this method in predicting treatment response and facilitating personalized treatment choices. Taken together, these results suggest 
the eSelect system is an efficient and reliable method of obtaining CTCs for anti-cancer drug sensitivity testing, and to the best of our knowledge, this is the first study that correlates drug sensitivity test results in ex vivo CTC cultures with clinical treatment outcomes in PDAC. Larger prospective trials are warranted to validate and expand upon these findings.

\section{Materials And Methods}

\section{Patients}

This observational study was approved by the institutional review board (IRB) of Taipei Medical University Hospital prior to initiation, and was conducted in accordance with institutional and local regulations, Good Clinical Practice (GCP), and the Declaration of Helsinki. Written informed consent was obtained from all patients prior to enrollment in the study. A total of 31 pathologically confirmed PDAC patients indicated for systemic treatment were enrolled from August 2018 to September 2020. Eligible patients were $\geq 20$ years old and were indicated for systemic treatment, with Karnofsky Performance Score $(K P S) \geq 70$, at least one measurable lesion, and life expectancy of more than 3 months. Age, sex, tumor staging, treatments, and clinical response were collected for each patient. Tumor staging was performed in accordance with the American Joint Committee on Cancer (AJCC) Cancer Staging Manual, Eighth Edition [18], and clinical response was evaluated using Response Evaluation Criteria in Solid Tumors (RECIST) version 1.1 [19] at the time of blood sampling and 3 months later.

\section{CTC Extraction and Organoid Expansion}

Liquid biopsies consisting of a 20-mL sample of peripheral venous blood were collected from each patient, using K2EDTA Vacutainers ${ }^{\circledR}$ (BD Bioscience, Franklin Lakes NJ, USA). A total of 41 liquid biopsies were collected, from which the peripheral blood mononuclear cell (PBMC) fraction containing CTCs was extracted using Ficoll-Paque centrifugation as previously described [17, 20]. Cells were then seeded onto a substrate of binary colloidal crystal (BCC) containing silica and polymethyl methacrylate (PMMA), and cultured in the eSelect system for 3 weeks, with replacement of culture medium every 4 days. Organoid expansion was monitored by visual microcopy, and the presence of CTCs was confirmed by EpCAM and CD45 immunofluorescence staining in a procedure described previously [17]. Patients for whom CTC-derived organoid expansion was unsuccessful were denoted as "failed expansion."

\section{Drug Sensitivity Profiling}

Expanded CTC-derived organoids were resuspended in culture medium, then divided into aliquots and loaded to 96-well plates for drug sensitivity assays. A PDAC-specific drug panel of nine drugs, including gemcitabine, 5-fluorouracil (5-FU), erlotinib, irinotecan, olaparib, oxaliplatin, paclitaxel, palbociclib, and trametinib, was tested against CTC cultures at clinically relevant concentrations [21]. Drug treatments were respectively added to each well, after which cultures were continued for 1 week. All tests were conducted in triplicate for each drug. At the end of the assay period, viable cell counts were measured using cytosolic adenosine triphosphate (ATP) abundance (CellTiter Glo, Promega, Madison WI, USA) via a 
luminometer (Promega). The results were normalized to untreated control groups to derive relative cell viability. Relative cell viability percentages for each drug were reported to the attending oncologist for each patient once available, usually around 6 weeks after liquid biopsies were obtained. The following threshold percentages were established following analysis of a receiver operating characteristic (ROC) curve using Youden's index: for relative cell viability ranging from $0.0-29.9 \%$, a score of 4 was given; for relative cell viability of $30.0-69.9 \%$, a score of 1 was given; and for relative cell viability of $70.0-100.0 \%$, a score of 0 was given. The CTS score was defined as the sum of all scores for drugs received by patients for a duration of more than 6 weeks in the 3 months before or after liquid biopsies were obtained. In addition, a binary drug sensitivity variable (e) was also assessed for all patients, and defined as positive (e+) if patients received treatment with any drug that had $<50 \%$ relative cell viability in drug sensitivity tests, and negative $(e)$ if patient treatments all had $\geq 50 \%$ relative cell viability. A drug was defined as being "unmatched" if the patient received the drug but there was no corresponding drug sensitivity test result available. Radiotherapy to the target lesion was defined as an unmatched drug in patients that received this treatment during the defined period. For patients with failed expansion or who only received unmatched drugs during the 3 months before or after liquid biopsies were obtained, CTS and $e$ status were assigned as "N/A" (not applicable).

\section{Clinical Response Assessment}

RECIST 1.1 criteria [19] was applied to evaluate tumor response. Medical images from patients obtained $3 \pm 1$ months prior to, within 1 month, and $3 \pm 1$ months after liquid biopsies were obtained, and were respectively defined as Image(-1), Image(0), and Image(1) (Fig. 1). Clinical response was reviewed independently by 2 gastrointestinal radiologists, 2 radiation oncologists, and 1 gastrointestinal oncologist blinded to patient medications and drug sensitivity test results, and scored as complete response (CR), partial response (PR), stable disease (SD), or progressive disease (PD) [19].

\section{Correlating Drug Sensitivity Profiles with Clinical Response}

Drugs received by patients for a duration of more than 6 weeks in the 3 months before or after liquid biopsies were obtained were included for prospective utility analysis between CTC-derived organoid drug sensitivity (CTS, e) and clinical treatment responses (CR, PR, SD, PD; Fig. 1). In the prospective utility analysis, to ascertain the predictive capability of drug sensitivity profiles, CTS ( $<4$ vs. $\geq 4$ ) was compared against clinical response (SD + PD vs. CR + PR) to later treatments after liquid biopsy, while e status (e+ vs. $e$ ) was compared against a binary clinical benefit variable $(c, c+v s . c)$, with $c+$ defined as $\mathrm{CR}$, $\mathrm{PR}$, or $\mathrm{SD}$, and $c$ - defined as PD.

\section{Statistical Analysis}

A point-biserial correlation test was utilized, and all statistical tests were performed with $\mathrm{R}$ 3.3.1 (https://www.r-project.org/) and GraphPad Prism 7 (San Diego, CA, USA). Odds ratios were calculated for $2 \times 2$ contingency tables, using the Haldane-Anscombe correction of adding 0.5 to all cells if there was at 
least one zero cell. Two-tailed Fisher's exact test was separately used to calculate $P$ value and assess significance, with statistical significance defined as $\mathrm{P}<0.05$.

\section{Results}

\section{Isolation and Expansion of CTCs}

A total of 41 fresh liquid biopsies derived from 31 enrolled patients were processed between 2018 and 2020 (Fig. 1). At Day 0, PBMCs with enriched CTCs were seeded to three-dimensional 24-well plates, with variable size and morphology. At Days 7 to 28 , cells were observed to become circular in shape and larger in size, with signature morphologies, eventually forming organoids (Fig. 2A). After 3 weeks of culturing, CTCs in the organoids were identified by EpCAM and CD45 immunofluorescence staining (Fig. 2B), with EpCAM-positive and CD45-negative cells considered to be CTCs in accordance with current laboratory standards [11]. Following confirmation with visual microscopy and immunostaining, it was ascertained that CTC-derived organoids were successfully cultured from $87.8 \%(36 / 41)$ of biopsies, with sufficient cells to conduct screening for 9 treatments in triplicate. Expanded CTC-derived organoids were resuspended in culture medium, and aliquots were placed into 96-well culture plates for drug sensitivity profiling.

\section{Patient Demographics}

This study enrolled 31 PDAC patients with pathologically confirmed disease between August 2018 to September 2020, with patient demographics of the tested liquid biopsies presented in Table 1. Slightly more than half $(53.7 \%)$ of the patients were female. The mean age was 63.0 years, with $39.0 \%$ of patients aged over 65 years. Most (87.8\%) patients presented with Stage 4 disease. A total of 41 liquid biopsies were collected from patients, and their profiles are presented in Table 2. CTC expansion failed for 5 liquid biopsies (failed expansion). Results from 36 liquid biopsies were included in the final prospective utility analysis conducted with CTS or $e$ status against clinical response or clinical benefit (c)(Fig. 3). 
Table 1

Patient demographics of liquid biopsies tested

\begin{tabular}{|c|c|c|}
\hline & \multicolumn{2}{|c|}{41 liquid biopsies taken from 31 enrolled patients } \\
\hline Mean age, years & \multicolumn{2}{|l|}{63.0} \\
\hline Age > 65 years, $n(\%)$ & \multicolumn{2}{|l|}{$16(39.0 \%)$} \\
\hline Female, n (\%) & \multicolumn{2}{|l|}{$22(53.7 \%)$} \\
\hline \multicolumn{3}{|c|}{ Cancer staging at time of liquid biopsy } \\
\hline Stage $4, \mathrm{n}(\%)$ & \multicolumn{2}{|l|}{$36(87.8 \%)$} \\
\hline Stage $3, \mathrm{n}(\%)$ & \multicolumn{2}{|l|}{$4(9.8 \%)$} \\
\hline Stage $2, \mathrm{n}(\%)$ & \multicolumn{2}{|l|}{$1(2.4 \%)$} \\
\hline \multirow[t]{2}{*}{ Treatments } & \multirow{2}{*}{$\begin{array}{l}3 \text { months prior to } \\
\text { liquid biopsy }\end{array}$} & 3 months after \\
\hline & & liquid biopsy \\
\hline 5-fluorouracil, n (\%) & \multicolumn{2}{|l|}{18 (43.9\%) } \\
\hline Erlotinib, n (\%) & \multicolumn{2}{|l|}{$6(14.6 \%)$} \\
\hline Gemcitabine, n (\%) & \multicolumn{2}{|l|}{$15(36.6 \%)$} \\
\hline Olaparib, n (\%) & \multicolumn{2}{|l|}{$1(2.4 \%)$} \\
\hline Oxaliplatin, n (\%) & \multicolumn{2}{|l|}{$6(14.6 \%)$} \\
\hline Paclitaxel, n (\%) & \multicolumn{2}{|r|}{$11(26.8 \%)$} \\
\hline Palbociclib, n (\%) & \multicolumn{2}{|l|}{$0(0.0 \%)$} \\
\hline Trametinib, n (\%) & \multicolumn{2}{|l|}{$0(0.0 \%)$} \\
\hline Cisplatin, n (\%) & \multicolumn{2}{|l|}{$1(2.4 \%)$} \\
\hline CCRT, n (\%) & \multicolumn{2}{|l|}{$3(7.3 \%)$} \\
\hline Radiotherapy, n (\%) & \multicolumn{2}{|l|}{$8(19.5 \%)$} \\
\hline Others, n (\%) & \multicolumn{2}{|l|}{$14(34.1 \%)$} \\
\hline No treatment, $\mathrm{n}(\%)$ & \multicolumn{2}{|l|}{$3(7.3 \%)$} \\
\hline \multirow[t]{2}{*}{ Clinical response } & 3 months prior to & 3 months after \\
\hline & \multicolumn{2}{|l|}{ liquid biopsy } \\
\hline Complete response, $\mathrm{n}(\%)$ & $0(0.0 \%)$ & $0(0.0 \%)$ \\
\hline
\end{tabular}

CCRT, concurrent chemoradiation therapy; N/A, not applicable. 


\begin{tabular}{|lll|}
\hline & \multicolumn{2}{l|}{41 liquid biopsies taken from 31 enrolled patients } \\
\hline Partial response, $\mathrm{n}(\%)$ & $3(7.3 \%)$ & $4(9.76 \%)$ \\
\hline Stable disease, $\mathrm{n}(\%)$ & $9(22.0 \%)$ & $16(39.0 \%)$ \\
\hline Progressive disease, $\mathrm{n}(\%)$ & $29(70.7 \%)$ & $20(48.78 \%)$ \\
\hline N/A, n (\%) & $0(0.0 \%)$ & $1(2.4 \%)$ \\
\hline CCRT, concurrent chemoradiation therapy; N/A, not applicable.
\end{tabular}


Table 2

Profiles of 41 liquid biopsies collected from 31 patients

\begin{tabular}{|c|c|c|c|c|c|c|c|c|}
\hline \multirow{2}{*}{$\begin{array}{l}\text { Patient } \\
\text { No. }\end{array}$} & \multirow{2}{*}{ Sex } & \multirow[t]{2}{*}{ Age } & \multirow[t]{2}{*}{$\begin{array}{l}\text { AJCC } \\
\text { Stage }\end{array}$} & \multirow[t]{2}{*}{$\begin{array}{l}\text { CTC } \\
\text { Expansion }\end{array}$} & \multicolumn{2}{|c|}{$\begin{array}{l}3 \text { months prior to liquid } \\
\text { biopsy }\end{array}$} & \multicolumn{2}{|c|}{$\begin{array}{l}3 \text { months after liquid } \\
\text { biopsy }\end{array}$} \\
\hline & & & & & $\begin{array}{l}\text { CTS } \\
\text { (e) }\end{array}$ & $\begin{array}{l}\text { Clinical } \\
\text { Response }\end{array}$ & $\begin{array}{l}\text { CTS } \\
\text { (e) }\end{array}$ & $\begin{array}{l}\text { Clinical } \\
\text { Response }\end{array}$ \\
\hline \multirow[t]{3}{*}{01} & $\mathrm{~F}$ & 61 & 4 & $\mathrm{~N}$ & N/A & $\begin{array}{l}\text { Progressive } \\
\text { Disease }\end{array}$ & N/A & $\begin{array}{l}\text { Partial } \\
\text { Response }\end{array}$ \\
\hline & & 61 & 4 & $\mathrm{Y}$ & $0(-)$ & $\begin{array}{l}\text { Progressive } \\
\text { Disease }\end{array}$ & $1(-)$ & $\begin{array}{l}\text { Progressive } \\
\text { Disease }\end{array}$ \\
\hline & & 61 & 4 & $\mathrm{Y}$ & $2(+)$ & Stable Disease & $\mathrm{N} / \mathrm{A}$ & $\begin{array}{l}\text { Progressive } \\
\text { Disease }\end{array}$ \\
\hline \multirow[t]{3}{*}{02} & $\mathrm{~F}$ & 64 & 4 & $\mathrm{Y}$ & $4(+)$ & Stable Disease & $4(+)$ & $\begin{array}{l}\text { Stable } \\
\text { Disease }\end{array}$ \\
\hline & & 64 & 4 & $Y$ & $1(-)$ & $\begin{array}{l}\text { Progressive } \\
\text { Disease }\end{array}$ & $1(-)$ & $\begin{array}{l}\text { Stable } \\
\text { Disease }\end{array}$ \\
\hline & & 64 & 4 & Y & N/A & $\begin{array}{l}\text { Progressive } \\
\text { Disease }\end{array}$ & $5(+)$ & $\begin{array}{l}\text { Progressive } \\
\text { Disease }\end{array}$ \\
\hline 03 & $\mathrm{~F}$ & 50 & 4 & Y & N/A & $\begin{array}{l}\text { Progressive } \\
\text { Disease }\end{array}$ & $0(-)$ & $\begin{array}{l}\text { Progressive } \\
\text { Disease }\end{array}$ \\
\hline \multirow[t]{2}{*}{04} & $M$ & 77 & 4 & $Y$ & N/A & $\begin{array}{l}\text { Progressive } \\
\text { Disease }\end{array}$ & $0(-)$ & $\begin{array}{l}\text { Stable } \\
\text { Disease }\end{array}$ \\
\hline & & 79 & 4 & $\mathrm{Y}$ & $\mathrm{N} / \mathrm{A}$ & Stable Disease & N/A & $\begin{array}{l}\text { Progressive } \\
\text { Disease }\end{array}$ \\
\hline 05 & $M$ & 51 & 4 & $\mathrm{~N}$ & $\mathrm{~N} / \mathrm{A}$ & $\begin{array}{l}\text { Progressive } \\
\text { Disease }\end{array}$ & $\mathrm{N} / \mathrm{A}$ & $\begin{array}{l}\text { Progressive } \\
\text { Disease }\end{array}$ \\
\hline 06 & $\mathrm{M}$ & 64 & 4 & Y & $0(-)$ & Stable Disease & $0(-)$ & $\begin{array}{l}\text { Stable } \\
\text { Disease }\end{array}$ \\
\hline 07 & $\mathrm{~F}$ & 57 & 4 & $Y$ & $1(-)$ & $\begin{array}{l}\text { Progressive } \\
\text { Disease }\end{array}$ & $2(+)$ & $\begin{array}{l}\text { Stable } \\
\text { Disease }\end{array}$ \\
\hline 08 & $\mathrm{~F}$ & 55 & 4 & $\mathrm{Y}$ & $1(-)$ & $\begin{array}{l}\text { Progressive } \\
\text { Disease }\end{array}$ & N/A & $\begin{array}{l}\text { Progressive } \\
\text { Disease }\end{array}$ \\
\hline 09 & $M$ & 67 & 4 & $\mathrm{~N}$ & $\mathrm{~N} / \mathrm{A}$ & $\begin{array}{l}\text { Progressive } \\
\text { Disease }\end{array}$ & N/A & N/A \\
\hline 10 & $M$ & 56 & 4 & $Y$ & N/A & $\begin{array}{l}\text { Progressive } \\
\text { Disease }\end{array}$ & $\begin{array}{l}0 \\
(\mathrm{~N} / \mathrm{A})\end{array}$ & $\begin{array}{l}\text { Stable } \\
\text { Disease }\end{array}$ \\
\hline 11 & $M$ & 60 & 4 & $\mathrm{Y}$ & $2(+)$ & $\begin{array}{l}\text { Progressive } \\
\text { Disease }\end{array}$ & $1(+)$ & $\begin{array}{l}\text { Stable } \\
\text { Disease }\end{array}$ \\
\hline
\end{tabular}

CTS, cytotoxicity score of received treatments; N/A, not applicable. 


\begin{tabular}{|c|c|c|c|c|c|c|c|c|}
\hline \multirow{2}{*}{$\begin{array}{l}\text { Patient } \\
\text { No. }\end{array}$} & \multirow{2}{*}{ Sex } & \multirow[t]{2}{*}{ Age } & \multirow[t]{2}{*}{$\begin{array}{l}\text { AJCC } \\
\text { Stage }\end{array}$} & \multirow[t]{2}{*}{$\begin{array}{l}\text { CTC } \\
\text { Expansion }\end{array}$} & \multicolumn{2}{|c|}{$\begin{array}{l}3 \text { months prior to liquid } \\
\text { biopsy }\end{array}$} & \multicolumn{2}{|c|}{$\begin{array}{l}3 \text { months after liquid } \\
\text { biopsy }\end{array}$} \\
\hline & & & & & $\begin{array}{l}\text { CTS } \\
\text { (e) }\end{array}$ & $\begin{array}{l}\text { Clinical } \\
\text { Response }\end{array}$ & $\begin{array}{l}\text { CTS } \\
\text { (e) }\end{array}$ & $\begin{array}{l}\text { Clinical } \\
\text { Response }\end{array}$ \\
\hline \multirow[t]{2}{*}{12} & $M$ & 57 & 4 & $Y$ & $3(+)$ & Stable Disease & $3(+)$ & $\begin{array}{l}\text { Progressive } \\
\text { Disease }\end{array}$ \\
\hline & & 58 & 4 & $\mathrm{Y}$ & $8(+)$ & $\begin{array}{l}\text { Progressive } \\
\text { Disease }\end{array}$ & $\begin{array}{l}0 \\
(\mathrm{~N} / \mathrm{A})\end{array}$ & $\begin{array}{l}\text { Progressive } \\
\text { Disease }\end{array}$ \\
\hline 13 & $F$ & 67 & 4 & $\mathrm{Y}$ & $1(-)$ & $\begin{array}{l}\text { Progressive } \\
\text { Disease }\end{array}$ & $1(-)$ & $\begin{array}{l}\text { Progressive } \\
\text { Disease }\end{array}$ \\
\hline 14 & $F$ & 60 & 4 & $\mathrm{Y}$ & $1(-)$ & $\begin{array}{l}\text { Partial } \\
\text { Response }\end{array}$ & $1(-)$ & $\begin{array}{l}\text { Stable } \\
\text { Disease }\end{array}$ \\
\hline 15 & $\mathrm{~F}$ & 77 & 2 & $Y$ & $1(+)$ & Stable Disease & $1(+)$ & $\begin{array}{l}\text { Stable } \\
\text { Disease }\end{array}$ \\
\hline 16 & $M$ & 48 & 4 & $\mathrm{Y}$ & $\mathrm{N} / \mathrm{A}$ & $\begin{array}{l}\text { Progressive } \\
\text { Disease }\end{array}$ & $4(+)$ & $\begin{array}{l}\text { Stable } \\
\text { Disease }\end{array}$ \\
\hline 17 & M & 70 & 4 & $\mathrm{~N}$ & $\mathrm{~N} / \mathrm{A}$ & $\begin{array}{l}\text { Progressive } \\
\text { Disease }\end{array}$ & $\mathrm{N} / \mathrm{A}$ & $\begin{array}{l}\text { Progressive } \\
\text { Disease }\end{array}$ \\
\hline 18 & $M$ & 68 & 4 & $\mathrm{Y}$ & $1(+)$ & $\begin{array}{l}\text { Progressive } \\
\text { Disease }\end{array}$ & $1(+)$ & $\begin{array}{l}\text { Progressive } \\
\text { Disease }\end{array}$ \\
\hline \multirow[t]{2}{*}{19} & $F$ & 49 & 4 & $\mathrm{Y}$ & $0(-)$ & $\begin{array}{l}\text { Progressive } \\
\text { Disease }\end{array}$ & $1(-)$ & $\begin{array}{l}\text { Progressive } \\
\text { Disease }\end{array}$ \\
\hline & & 49 & 4 & $\mathrm{Y}$ & $1(+)$ & $\begin{array}{l}\text { Progressive } \\
\text { Disease }\end{array}$ & $\mathrm{N} / \mathrm{A}$ & $\begin{array}{l}\text { Progressive } \\
\text { Disease }\end{array}$ \\
\hline 20 & $\mathrm{~F}$ & 83 & 3 & $\mathrm{Y}$ & $\mathrm{N} / \mathrm{A}$ & $\begin{array}{l}\text { Progressive } \\
\text { Disease }\end{array}$ & $1(-)$ & $\begin{array}{l}\text { Progressive } \\
\text { Disease }\end{array}$ \\
\hline 21 & $\mathrm{~F}$ & 63 & 4 & $\mathrm{Y}$ & $0(-)$ & $\begin{array}{l}\text { Partial } \\
\text { Response }\end{array}$ & $0(-)$ & $\begin{array}{l}\text { Stable } \\
\text { Disease }\end{array}$ \\
\hline 22 & $M$ & 84 & 3 & $Y$ & $\mathrm{~N} / \mathrm{A}$ & $\begin{array}{l}\text { Progressive } \\
\text { Disease }\end{array}$ & $\mathrm{N} / \mathrm{A}$ & $\begin{array}{l}\text { Progressive } \\
\text { Disease }\end{array}$ \\
\hline 23 & $\mathrm{~F}$ & 61 & 4 & $\mathrm{~N}$ & N/A & Stable Disease & $\mathrm{N} / \mathrm{A}$ & $\begin{array}{l}\text { Stable } \\
\text { Disease }\end{array}$ \\
\hline \multirow[t]{2}{*}{24} & $M$ & 70 & 3 & $Y$ & $\mathrm{~N} / \mathrm{A}$ & Stable Disease & $8(+)$ & $\begin{array}{l}\text { Progressive } \\
\text { Disease }\end{array}$ \\
\hline & & 70 & 4 & $\mathrm{Y}$ & $8(+)$ & $\begin{array}{l}\text { Progressive } \\
\text { Disease }\end{array}$ & $8(+)$ & $\begin{array}{l}\text { Stable } \\
\text { Disease }\end{array}$ \\
\hline 25 & $M$ & 79 & 4 & $\mathrm{Y}$ & $1(+)$ & $\begin{array}{l}\text { Progressive } \\
\text { Disease }\end{array}$ & $5(+)$ & $\begin{array}{l}\text { Progressive } \\
\text { Disease }\end{array}$ \\
\hline
\end{tabular}




\begin{tabular}{|c|c|c|c|c|c|c|c|c|}
\hline \multirow{2}{*}{$\begin{array}{l}\text { Patient } \\
\text { No. }\end{array}$} & \multirow{2}{*}{ Sex } & \multirow[t]{2}{*}{ Age } & \multirow[t]{2}{*}{$\begin{array}{l}\text { AJCC } \\
\text { Stage }\end{array}$} & \multirow[t]{2}{*}{$\begin{array}{l}\text { CTC } \\
\text { Expansion }\end{array}$} & \multicolumn{2}{|c|}{$\begin{array}{l}3 \text { months prior to liquid } \\
\text { biopsy }\end{array}$} & \multicolumn{2}{|c|}{$\begin{array}{l}3 \text { months after liquid } \\
\text { biopsy }\end{array}$} \\
\hline & & & & & $\begin{array}{l}\text { CTS } \\
\text { (e) }\end{array}$ & $\begin{array}{l}\text { Clinical } \\
\text { Response }\end{array}$ & $\begin{array}{l}\text { CTS } \\
\text { (e) }\end{array}$ & $\begin{array}{l}\text { Clinical } \\
\text { Response }\end{array}$ \\
\hline \multirow[t]{2}{*}{26} & $M$ & 71 & 4 & $Y$ & $1(+)$ & $\begin{array}{l}\text { Progressive } \\
\text { Disease }\end{array}$ & $1(+)$ & $\begin{array}{l}\text { Progressive } \\
\text { Disease }\end{array}$ \\
\hline & & 71 & 4 & $Y$ & $0(-)$ & $\begin{array}{l}\text { Progressive } \\
\text { Disease }\end{array}$ & $0(-)$ & $\begin{array}{l}\text { Progressive } \\
\text { Disease }\end{array}$ \\
\hline \multirow[t]{2}{*}{27} & $\mathrm{~F}$ & 49 & 3 & $Y$ & $\mathrm{~N} / \mathrm{A}$ & $\begin{array}{l}\text { Progressive } \\
\text { Disease }\end{array}$ & $4(+)$ & $\begin{array}{l}\text { Partial } \\
\text { Response }\end{array}$ \\
\hline & & 49 & 4 & $Y$ & $\mathrm{~N} / \mathrm{A}$ & $\begin{array}{l}\text { Progressive } \\
\text { Disease }\end{array}$ & N/A & $\begin{array}{l}\text { Progressive } \\
\text { Disease }\end{array}$ \\
\hline 28 & $\mathrm{~F}$ & 26 & 4 & $Y$ & $4(+)$ & $\begin{array}{l}\text { Progressive } \\
\text { Disease }\end{array}$ & $5(+)$ & $\begin{array}{l}\text { Stable } \\
\text { Disease }\end{array}$ \\
\hline 29 & $\mathrm{M}$ & 80 & 4 & Y & $4(+)$ & Stable Disease & $4(+)$ & $\begin{array}{l}\text { Stable } \\
\text { Disease }\end{array}$ \\
\hline 30 & $\mathrm{~F}$ & 70 & 4 & Y & $\mathrm{N} / \mathrm{A}$ & $\begin{array}{l}\text { Progressive } \\
\text { Disease }\end{array}$ & $5(+)$ & $\begin{array}{l}\text { Partial } \\
\text { Response }\end{array}$ \\
\hline 31 & $\mathrm{~F}$ & 63 & 4 & $Y$ & $5(+)$ & Stable Disease & $5(+)$ & $\begin{array}{l}\text { Partial } \\
\text { Response }\end{array}$ \\
\hline
\end{tabular}

\section{Drug Sensitivity Testing and Comparison with Clinical Response}

After 3 weeks of culturing, CTC-derived organoids were resuspended in culture medium and then aliquoted to 96-well plates, in order to assess sensitivity to a PDAC-specific panel of nine drugs, including 5-fluorouracil (5-FU), erlotinib, gemcitabine, irinotecan, olaparib, oxaliplatin, paclitaxel, palbociclib, and trametinib. All drug sensitivity assays were conducted in triplicate. Drugs were respectively applied at clinically relevant concentrations for a week, after which relative cell viability was assessed and CTS and e status were calculated. Lower cell viability following application of test treatments was indicative of greater drug sensitivity and better anti-tumor effect. Relative cell viability was used to calculate CTS and $e$ status for each patient both retrospectively and prospectively (Table 2). Prospective utility analysis was conducted to compare drug sensitivity results with treatments received by patients for at least 6 weeks in duration during the 3-month period after liquid biopsy, and the results showed significant association between CTS and clinical response $(P=0.0406$; Odds Ratio $(O R)=16.0588,95 \%$ confidence interval $(C I)$ : 0.7449-346.2114; Table 3). However, $e$ status did not correlate significantly with $c$ status in prospective utility analysis $(P=0.4410$; Table 4$)$. 
Prospective utility analysis of CTS and clinical response

\begin{tabular}{|c|c|c|c|c|}
\hline CTS & Progressive Disease & Stable Disease & Partial Response & $\begin{array}{l}\text { Complete } \\
\text { Response }\end{array}$ \\
\hline $\mathrm{N} / \mathrm{A}$ & 8 & 1 & 1 & 0 \\
\hline 0 & 3 & 4 & 0 & 0 \\
\hline 1 & 6 & 4 & 0 & 0 \\
\hline 2 & 0 & 1 & 0 & 0 \\
\hline 3 & 1 & 0 & 0 & 0 \\
\hline 4 & 0 & 3 & 1 & 0 \\
\hline 5 & 2 & 1 & 2 & 0 \\
\hline 6 & 0 & 0 & 0 & 0 \\
\hline 7 & 0 & 0 & 0 & 0 \\
\hline 8 & 1 & 1 & 0 & 0 \\
\hline 9 & 0 & 0 & 0 & 0 \\
\hline Utility & $\begin{array}{l}\text { Stable or Progressive } \\
\text { Disease }\end{array}$ & $\begin{array}{l}\text { Partial or Complete } \\
\text { Response }\end{array}$ & $\begin{array}{l}\text { Two-tailed Fisher's } \\
\text { exact test }\end{array}$ & $P=0.0406$ \\
\hline $\begin{array}{l}\text { CTS } \\
<4\end{array}$ & 19 & 0 & \multirow[t]{3}{*}{ Odds Ratio } & 16.0588 \\
\hline \multirow{2}{*}{$\begin{array}{l}\text { CTS } \\
\geq 4\end{array}$} & 8 & 3 & & $\begin{array}{l}\text { 95\% Cl: } 0.7449- \\
346.2114\end{array}$ \\
\hline & & & & $P=0.0764$ \\
\hline
\end{tabular}

Table 4

Prospective utility analysis of e status and clinical response

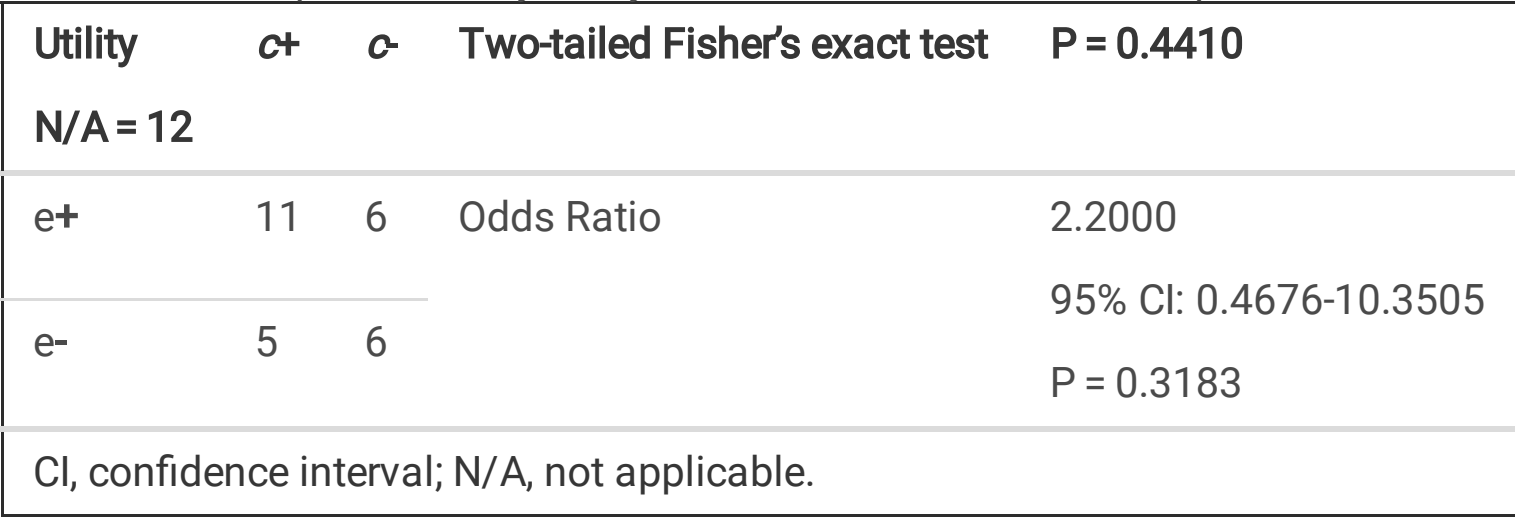

\section{Illustrative Case Study 1: Identifying Effective Treatments to Address Relapse}


Here we describe two representative cases to illustrate the potential application of this system in different clinical scenarios encountered in this study. A 61-year-old female patient presenting with locally advanced pancreatic cancer experienced intraperitoneal disease progression after concurrent chemoradiotherapy and maintenance weekly gemcitabine. She was enrolled in our study and CTC samples were obtained in October 2018. During this period, her clinical performance continued to deteriorate, and clinical images indicated progression of intraperitoneal diseases despite salvage treatment with three courses of FOLFIRI. CTC sensitivity profiling showed resistance to 5-FU, oxaliplatin, paclitaxel, and irinotecan, and the findings were consistent with the clinical disease course. However, erlotinib was found to suppress CTC proliferation with viability value at $27.8 \%$. She received erlotinib combined with gemcitabine at the discretion of the treating physician from December 2018, and enjoyed a clinical response for 4 months with improved performance status and decreased serum level of CA199. Her primary tumor biopsy was retrospectively analyzed, and Sanger sequencing revealed an epidermal growth factor receptor (EGFR) exon 20 mutation. It has been reported that erlotinib in combination with gemcitabine is active in patients with EGFR-mutated pancreatic cancer, with disease control rates of $64 \%$ [22]. A combination of cell sensitivity profiling and tumor sequencing can provide response biomarkers, as has been observed in this case.

\section{Illustrative Case Study 2: Phenotype Correlation with Unexpected Genotype}

A 57-year-old male patient was admitted for occult gastrointestinal bleeding on May 09, 2019, and was subsequently diagnosed with metastatic pancreatic cancer. Computer tomography (CT) scanning identified a 4.7-cm pancreatic tail tumor and 15 liver metastases. The patient's older sister was previously diagnosed with ovarian cancer and passed away at the age of 65. Radiotherapy (44 Gy/20 Fx) was initiated for the patient on May 16,2019, and the patient also received 12 cycles of chemotherapy with FOLFIRINOX beginning on May 28, 2019. In August 2019, an abdominal CT scan revealed SD for the pancreatic mass and PR for the liver metastases. However, a follow-up CT scan in November 2019 indicated PD in the pancreatic tumor, together with liver, bone, and peritoneal seeding. A 20-mL liquid biopsy was collected from the patient in October 2019 prior to the follow-up scan, and CTC-derived organoid expansion was successfully achieved in the eSelect system. Drug sensitivity testing indicated that relative cell viability following olaparib treatment was $16.7 \%$. Genetic testing was subsequently conducted on a biopsy taken from this patient in January 2020, and the results showed that the patient carried mutations in both the ATM (ataxia telangiectasia mutated) and BRIP1 (BRCA1-interacting protein 1) genes. ATM and BRIP1 work with BRCA1 (breast cancer 1) to repair DNA damage through the homologous recombination pathway, and tumor cells with defects in this pathway are dependent on the polyadenosine 5'-diphosphoribose [poly-(ADP-ribose)] polymerization (PARP) pathway for DNA repair. Olaparib is a PARP inhibitor that can block this pathway and disrupt DNA repair in tumor cells. Considering that mutations in $B R C A 1$ and the homologous recombination pathway are rarely seen in PDAC, with a prevalence rate of $<3 \%$ [23], the potential effectiveness of olaparib as predicted by the drug sensitivity test and subsequently corroborated in the genetic analysis report suggest that the eSelect 
system has the potential to reveal the presence of key mutations or other genetic features that would increase the efficacy of targeted treatments.

\section{Discussion}

In this study, we showed that eSelect, a biomimetic cell culture system for CTC isolation and ex vivo expansion developed by our lab, achieved $87.8 \%$ CTC culture success rates from PDAC patient liquid biopsies, and prospective utility analysis indicated that drug sensitivity assay results obtained from CTCderived organoid cultures correlated with clinical response to treatment in patients (Table 3 and Table 4). To the best of our knowledge, this is the first study to describe an association between drug sensitivity testing results on CTC-derived organoids and clinical treatment responses in PDAC patients. Moreover, the culture efficiency of $87.8 \%$ and the relatively short expansion time of 3-4 weeks compares favorably with previous studies [14-16], which reported efficiency rates of less than $20 \%$ and culturing times of 6 months or more. Furthermore, in contrast to patient-derived organoids (PDOs) derived from tumor biopsies [24, 25], less invasive methods are needed to obtain CTC-derived organoids, and this has particular relevance for PDAC, considering that approximately $80-90 \%$ of patients have unresectable disease at initial diagnosis [2]. In addition, extraction of CTCs directly from blood samples prevents both the loss of non-epithelial CTCs through antigen-based detection and the elimination of smaller and more deformable CTCs when physical techniques are used. Importantly, the eSelect system can also conduct high-throughput drug sensitivity screening for several different anti-cancer treatments at the same time, and this has the potential to be used for early-stage cancer detection, optimal treatment selection, treatment efficacy monitoring, and effective treatment screening in case of relapse or treatment failure. However, it is true that CTC-based systems also face a number of challenges. Previous studies have shown that CTCs can be captured and enriched, using epithelial cell markers to trap specific subpopulations of CTCs [26, 27]; but as epithelial-to mesenchymal transition often occurs in CTCs at a high frequency, CTCs often present in epithelial, mesenchymal, and mixed stages [28]. A previous study has indicated that both epithelial and mesenchymal markers were detected in breast cancer CTCs, with a higher ratio of mesenchymal-positive cells corresponding to the triple-negative subtype, progressive disease, and CTC clusters [29]. After enrichment, there is currently no method to eliminate any subpopulation of CTCs in order to maintain the heterogeneity of CTCs in the system, and whether this heterogeneity is important for the correlation between drug sensitivity testing results and clinical treatment responses remains to be determined.

Prospective utility analysis indicated significant correlations with clinical response for CTS (Table 3), suggesting that it is possible to predict treatment outcomes in advanced PDAC patients through prior testing of anti-cancer drugs in CTC-derived organoid cultures expanded ex vivo. At the very least, these findings show that the eSelect system can serve to eliminate ineffective treatments without having to go through a costly and time-consuming process of trial-and-error in patients. In an aggressive cancer such as advanced PDAC, the time and effort saved from this predictive information could bring about significant improvements in patient outcomes. 
This study has several limitations. Firstly, treatment heterogeneity and frequent ineligibility for planned analyses are inherent features of observational study designs in biomarker research. Since only a small number of patients were involved in this proof-of-concept biomarker study, a larger randomized controlled trial will be needed to validate these findings. Secondly, the CTC-derived organoids in the eSelect system cannot recapitulate the vascular and immune tumor microenvironment. This may be a relevant issue when seeking to predict the treatment response of drugs that depend on these stromal components. Thirdly, the effects of paclitaxel and irinotecan nanoparticle formulations were approximated using free drugs. This may lead to false negative prediction and may underestimate the diagnostic accuracy of the CTS. Lastly, it was not possible in this research protocol to compare the results of genetic and/or phenotypic analysis of CTC-derived organoids with those from concurrent tumor biopsies. Such information would be invaluable to disclose tumor evolution in the process of CTC formation, and provide further insights into the biological nature of CTC-derived organoids.

\section{Conclusions}

In this study, we describe an efficient and reliable method of expanding CTCs from liquid biopsies in PDAC patients, as well as the results of ex vivo drug sensitivity testing performed on these CTC-derived organoids. This proof-of-concept data reveals potential for the development of a non-invasive biomarker system that can predict treatment response, and larger prospective controlled studies are warranted for further validation.

\section{Abbreviations}

5-FU, 5-fluorouracil; AJCC, American Joint Committee on Cancer; ATP, adenosine triphosphate; ATM, ataxia telangiectasia mutated; BCC, binary colloidal crystal; BRCA1, breast cancer 1; BRIP1, BRCA1interacting protein 1 ; CCRT, concurrent chemoradiation therapy; $\mathrm{Cl}$, confidence interval; $\mathrm{CR}$, complete response; $\mathrm{CT}$, computer tomography; $\mathrm{CTC}$, circulating tumor cell; $\mathrm{CTS}$, cytotoxicity score; ECOG, Eastern Cooperative Oncology Group; GCP, Good Clinical Practice; IRB, institutional review board; KFS, Karnofsky Performance Score; N/A, not available; OR, Odds Ratio; PARP, polyadenosine 5'-diphosphoribose [poly(ADP-ribose)] polymerization; PBMC, peripheral blood mononuclear cells; PD, progressive disease; PDAC, pancreatic ductal adenocarcinoma; PDO, patient-derived organoid; PFS, progression-free survival; PMMA, polymethyl methacrylate; PR, partial response; RECIST, Response Evaluation Criteria In Solid Tumors; $\mathrm{ROC}$, receiver operating characteristic; SD, stable disease.

\section{Declarations}

\section{Acknowledgements}

This work was supported by the TMU Research Center of Cancer Translational Medicine from the Featured Areas Research Center Program within the Higher Education Sprout Project framework by the Ministry of Education in Taiwan. We thank our patients for agreeing to participate in this study. 


\section{Authors' contributions}

YHW initiated the study, collected data and wrote the manuscript. YPH, NCC, RCL, YMK, and JYW reviewed clinical courses blinded to the test results. CPL, YC, YMS, SEW, SCC, SHY, KDL, HET, JRT, RHT, and JFC cared for patients and provided advice. SHL, SMH, and YHC conducted statistical analysis and checked statistical results. TB, PYW, and YJC developed key research techniques and oversaw the study processes. LSL initiated the study, cared for patients, analyzed the data, developed key research techniques, oversaw the study processes, and wrote the manuscript. All authors reviewed and approved of the final manuscript.

\section{Funding}

This work was financially supported by the TMU Research Center of Cancer Translational Medicine from the Featured Areas Research Center Program within the framework of the Higher Education Sprout Project by the Ministry of Education in Taiwan. (Grant numbers: DP2-110-21121-03-C-06-01, DP2-110-21121-03C-06-02, DP2-110-21121-03-C-06-03, DP2-109-21121-03-C-06-01, DP2-109-21121-03-C-06-02, DP2-10921121-03-C-06-03.) This research was also partially supported by the Ministry of Science and Technology (Grant numbers: MOST-110-2320-B-038-056, MOST-110-2314-B-038-138, MOST-109-2314-B-038-122, MOST-109-2314-B-038-141, MOST-109-2635-B-038-001, MOST-109-2314-B-038-072, MOST 106-2320-B038-039), Taipei Medical University (Grant number: TMU105-AE1-B13) and National Health Research Institutes (Grant number: NHRI-EX109-10713EI). The funders had no role in the design of the study, collection, analysis, and interpretation of the data, or the writing and publication of this manuscript.

\section{Availability of data and materials}

The datasets from this study are available upon reasonable request to the corresponding author, subject to approval by the IRB of Taipei Medical University Hospital, Taipei, Taiwan.

\section{Ethics approval and consent to participate}

This observational study was approved by the IRB of Taipei Medical University, Taipei, Taiwan. All patients provided written informed consent prior to enrollment.

\section{Competing interests}

The authors declare that they have no competing interests.

\section{References}

1. Pietri E, Balsano R, Coriano M, Gelsomino F, Leonardi F, Bui S, et al. The implication of liquid biopsies to predict chemoresistance in pancreatic cancer. Cancer Drug Resist. 2021;4: [ePub online ahead of print]. DOI:10.20517/cdr.2021.01. 
2. Elsayed M, Abdelrahim M. The Latest Advancement in Pancreatic Ductal Adenocarcinoma Therapy: A Review Article for the Latest Guidelines and Novel Therapies. Biomedicines. 2021;9(4):389.

3. Rawla P, Sunkara T, Gaduputi V. Epidemiology of Pancreatic Cancer: Global Trends, Etiology and Risk Factors. World J Oncol. 2019;10(1):10-27.

4. Sung H, Ferlay J, Siegel RL, Laversanne M, Soerjomataram I, Jemal A, et al. CA Global cancer statistics 2020, GLOBOCAN estimates of incidence and mortality worldwide for 36 cancers in 185 countries. CA Cancer J Clin. 2021;71(3):209-49.

5. Sharma J, Shum E, Chau V, Paucar D, Cheng H, Halmos B. The Evolving Role of Biomarkers in Personalized Lung Cancer Therapy. Respiration. 2017;93:1-14.

6. Bownes RJ, Turnbull AK, Martinez-Perez C, Cameron DA, Sims AH, Oikonomidou O. On-treatment biomarkers can improve prediction of response to neoadjuvant chemotherapy in breast cancer. Breast Cancer Res. 2019;21:73.

7. Golan T, Hammel P, Reni M, Van Cutsem E, Macarulla T, Hall MJ, et al. Maintenance Olaparib for Germline BRCA-Mutated Metastatic Pancreatic Cancer. N Engl J Med. 2019;381(4):317-27.

8. Park W, Wong W, Yu KH, Varghese AM, Riaz N, Balachandran VP, et al. Homologous recombination deficiency (HRD): A biomarker for first-line (1L) platinum in advanced pancreatic ductal adenocarcinoma (PDAC). J Clin Oncol. 2019;37(15_suppl):4132-2.

9. Wong W, Raufi AG, Safyan RA, Bates SE, Manji GA. BRCA Mutations in Pancreas Cancer: Spectrum, Current Management, Challenges and Future Prospects. Cancer Manag Res. 2020;12:2731-42.

10. Lee JS, Park SS, Lee YK, Norton JA, Jeffrey SS. Liquid biopsy in pancreatic ductal adenocarcinoma: current status of circulating tumor cells and circulating tumor DNA. Mol Oncol. 2019;13(8):1623-50.

11. Martini V, Timme-Bronsert S, Fichtner-Feigl S, Hoeppner J, Kulemann B. Circulating Tumor Cells in Pancreatic Cancer: Current Perspectives. Cancers (Basel). 2019;11(11):1659.

12. Grillet $F$, Bayet E, Villeronce $O$, Zappia L, Lagerqvist EL, Lunke $S$, et al. Circulating tumour cells from patients with colorectal cancer have cancer stem cell hallmarks in ex vivo culture. Gut. 2017;66(10):1802-10.

13. Ramzy GM, Koessler T, Ducrey E, McKee T, Ris F, Buchs N, et al. Patient-Derived In Vitro Models for Drug Discovery in Colorectal Carcinoma. Cancers (Basel). 2020;12(6):1423.

14. Yu M, Bardia A, Aceto N, Bersani F, Madden MW, Donaldson MC, et al. Cancer therapy. Ex vivo culture of circulating breast tumor cells for individualized testing of drug susceptibility. Science. 2014;345(6193):216-20.

15. Gao D, Vela I, Sboner A, laquinta PJ, Karthaus WR, Gopalan A, et al. Organoid cultures derived from patients with advanced prostate cancer. Cell. 2014;159(1):176-87.

16. Praharaj PP, Bhutia SK, Nagrath S, Bitting RL, Deep G. Circulating tumor cell-derived organoids: Current challenges and promises in medical research and precision medicine. Biochim Biophys Acta Rev Cancer. 2018;1869(2):117-27. 
17. Lee HL, Chiou JF, Wang PY, Lu LS, Shen CN, Hsu HL, et al. Ex Vivo Expansion and Drug Sensitivity Profiling of Circulating Tumor Cells from Patients with Small Cell Lung Cancer. Cancers (Basel). 2020;12:3394.

18. Amin MB, Edge SB, Greene FL, Byrd DR, Brookland RK, Washington MK et al, eds. AJCC Cancer Staging Manual, 8th Edition, 2017; Springer International Publishing: New York, USA.

19. Eisenhauer EA, Therasse P, Bogaerts J, Schwartz LH, Sargent D, Ford R, et al. New response evaluation criteria in solid tumours: revised RECIST guideline (version 1.1). Eur $\mathrm{J}$ Cancer. 2009;45(2):228-47.

20. Lu SH, Tsai WS, Chang YH, Chou TY, Pang ST, Lin PH, et al. Identifying cancer origin using circulating tumor cells. Cancer Biol Ther. 2016;17(4):430-8.

21. Liston DR, Davis M. Clinically Relevant Concentrations of Anticancer Drugs: A Guide for Nonclinical Studies. Clin Cancer Res. 2017;23(14):3489-98.

22. Wang JP, Wu CY, Yeh YC, Shyr YM, Wu YY, Kuo CY, et al. Erlotinib is effective in pancreatic cancer with epidermal growth factor receptor mutations: a randomized, open-label, prospective trial. Oncotarget. 2015;6(20):18162-73.

23. Takeuchi S, Doi M, Ikari N, Yamamoto M, Furukawa T. Mutations in BRCA1, BRCA2, and PALB2, and a panel of 50 cancer-associated genes in pancreatic ductal adenocarcinoma. Sci Rep. 2018;8(1):8105.

24. Khoo BL, Lee SC, Kumar P, Tan TZ, Warkiani ME, Ow SG, et al. Short-term expansion of breast circulating cancer cells predicts response to anti-cancer therapy. Oncotarget. 2015;6(17):15578-93.

25. Hou HW, Warkiani ME, Khoo BL, Li ZR, Soo RA, Tan DS, et al. Isolation and retrieval of circulating tumor cells using centrifugal forces. Sci Rep. 2013;3:1259.

26. Nagrath S, Sequist LV, Maheswaran S, Bell DW, Irimia D, Ulkus L, et al. Isolation of rare circulating tumour cells in cancer patients by microchip technology. Nature. 2007;450(7173):1235-9.

27. Riethdorf S, Fritsche H, Muller V, Rau T, Schindlbeck C, Rack B, et al. Detection of circulating tumor cells in peripheral blood of patients with metastatic breast cancer: a validation study of the CellSearch system. Clin Cancer Res. 2007;13(3):920-8.

28. Thiery JP. Epithelial-mesenchymal transitions in tumour progression. Nat Rev Cancer. 2002;2(6):442-54.

29. Yu M, Bardia A, Wittner BS, Stott SL, Smas ME, Ting DT, et al. Circulating breast tumor cells exhibit dynamic changes in epithelial and mesenchymal composition. Science. 2013;339(6119):580-4.

\section{Figures}




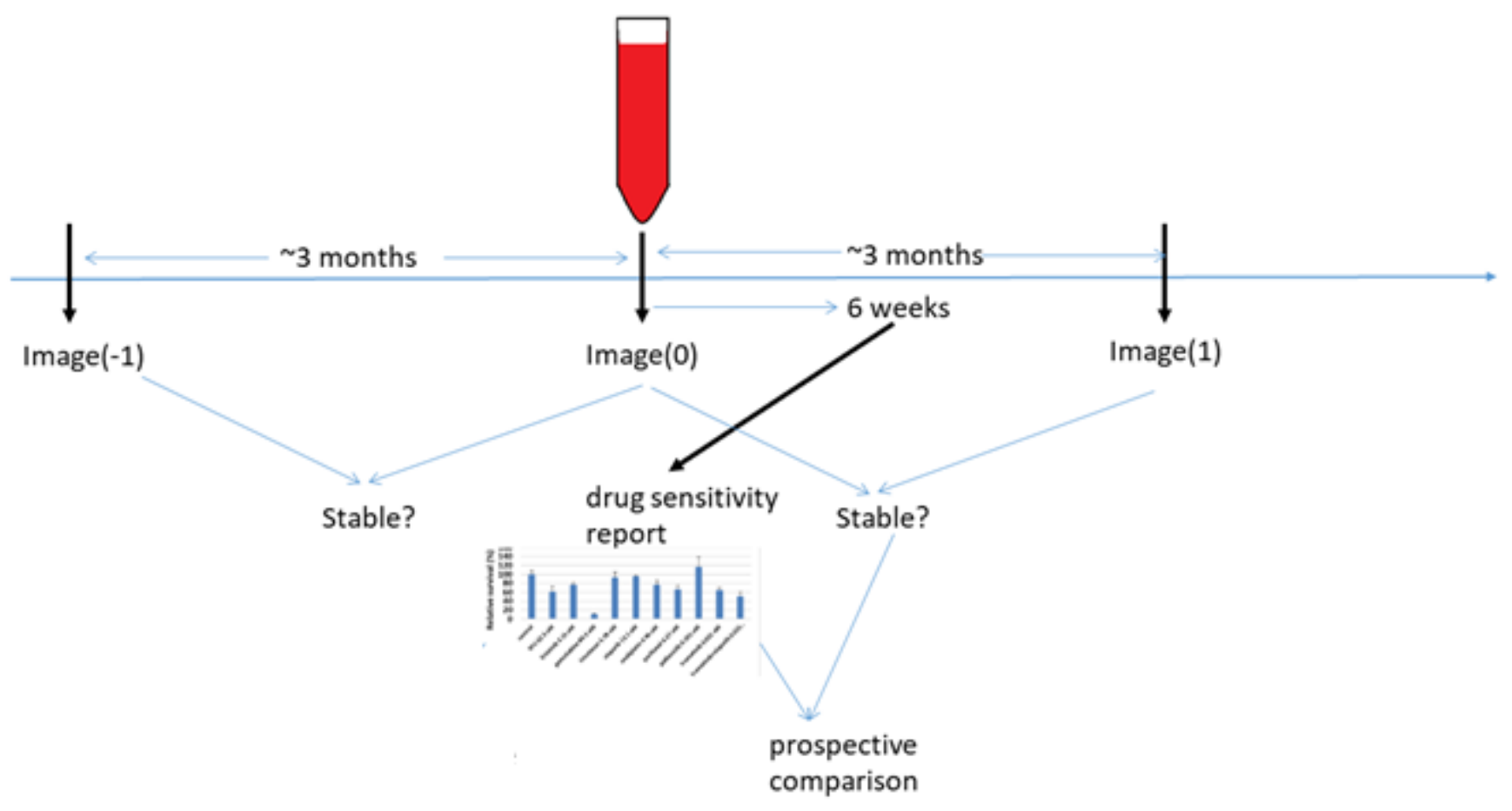

\section{Figure 1}

Flowchart of experimental procedures conducted for enrolled patients. A liquid biopsy was taken from patients, from which CTCs were isolated and expanded ex vivo to form CTC-derived organoid cultures, which were then tested for sensitivity to a panel of PDAC-specific drugs. Drug sensitivity test results were compared with clinical response to treatment(s) received for a minimum of 6 weeks, in the 3-month period after liquid biopsy (prospective utility analysis). 
(A)
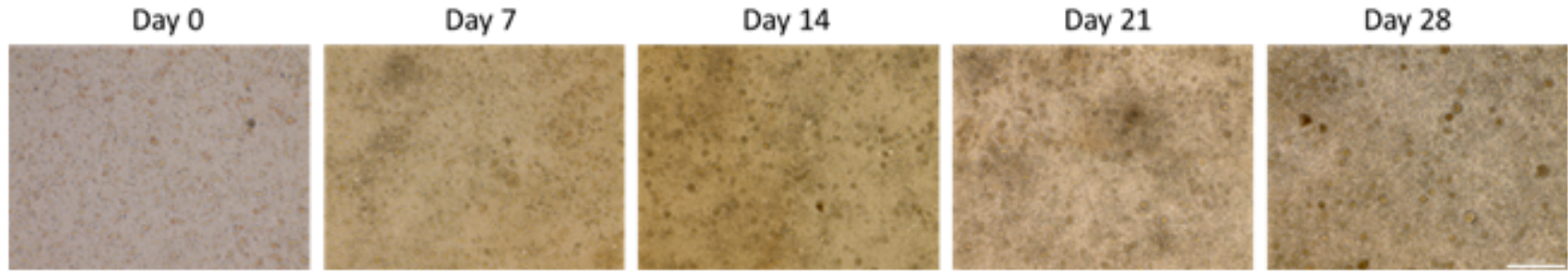

(B)

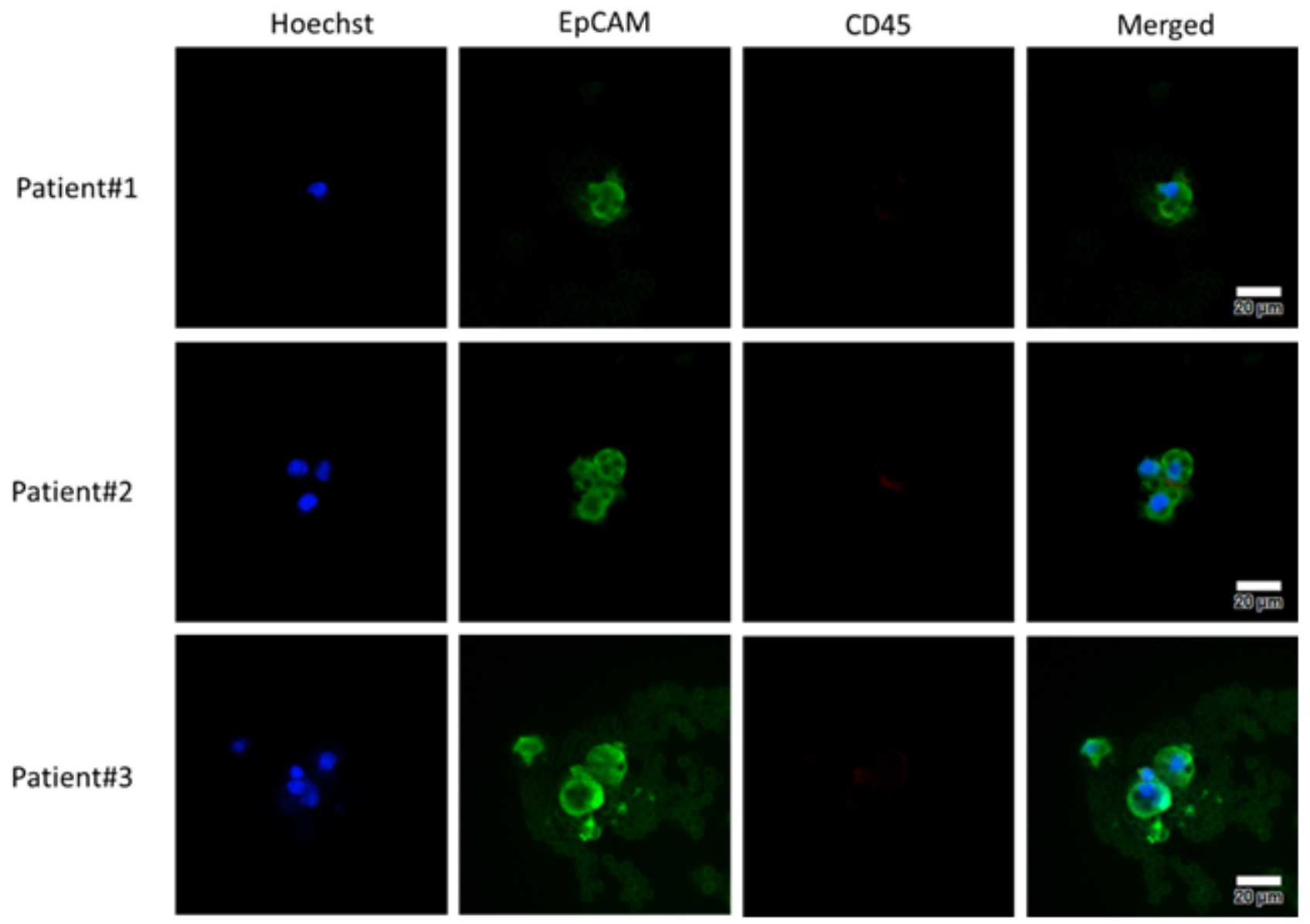

Figure 2

CTC-derived organoids expanded ex vivo from PDAC patient CTCs in liquid biopsies. (A) Representative bright field images of CTC-derived organoids. Scale bar: $100 \mu \mathrm{m}$. (B) Representative EpCam, CD 45, and Hoechst staining results from three patients to confirm the presence of CTCs. Scale bar: $20 \mu \mathrm{m}$. 


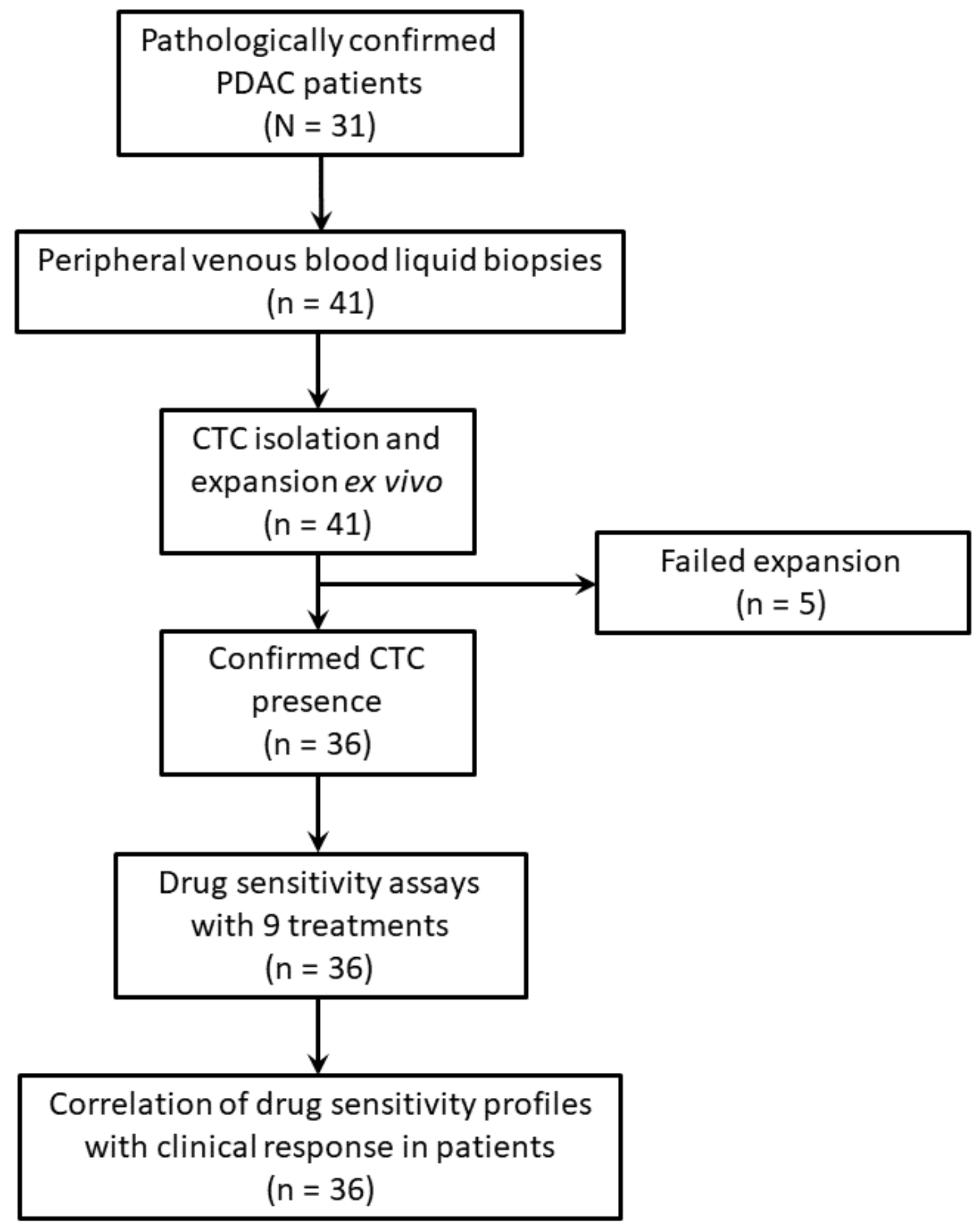

Figure 3

Overview of patient enrollment and CTC extraction and expansion from liquid biopsies. 